HEART REVIEW

The pressure wire in practice

Lucy J Blows, Simon R Redwood

Fractional flow reserve (FFR) is increasingly used to evaluate the functional significance of epicardial coronary disease. When compared with non-invasive techniques this index has superior sensitivity and specificity, is largely independent of prevailing haemodynamic conditions and has an unequivocal normal value. Furthermore, it can be used at the time of invasive coronary assessment. FFR measurements are invaluable for evaluation of intermediate coronary lesions but clinical decisions must be based on accurate measurements and an understanding of the limitations of the data supporting its use. This article identifies procedural and clinical issues pertaining to measurement of FFR and identifies potential piffalls and situations where interpretation can be difficult; for example, the presence of serial stenoses and left main stem disease, its use in specific conditions such as diffuse epicardial disease, microvascular abnormalities, left ventricular dysfunction and acute coronary syndromes.

See end of article for authors' affiliations

......................

Correspondence to: Dr S Redwood,

Cardiothoracic Unit, St

Thomas' Hospital, Lambeth

Palace Road, London SE 1

7EH, UK; simon.redwood@

gstt.nhs.uk

Accepted 14 April 2006

Published Online First

2 May 2006 t is well established that coronary angiography alone correlates poorly with the functional importance of epicardial coronary disease. Patients are often found to have lesions that seem to be intermediate in severity and have not had ischaemia documented with prior non-invasive testing that can be attributed to a specific lesion. This may lead to treating a lesion without benefit to the patient with the short and long-term risks inherent in that strategy or alternatively, inappropriately withholding revascularisation. In such situations, the use of a pressure-sensor-tipped guidewire has dramatically improved practice. The expansion of the use of this technology is reflected in the British Cardiovascular Intervention Society (BCIS) audit data. In 2004, according to BCIS figures, there was an increase of $30.5 \%$ in the use of the pressure wire from 897 to 1281 procedures.

Historically, a number of physiological measurements have been proposed to determine the importance of an individual coronary lesion, but these have largely been based on measurements of coronary flow with its inherent in vivo difficulties and dependence on prevailing haemodynamic conditions. Although these are of great value in a research setting, their clinical use is limited. ${ }^{1}$

The fractional flow reserve (FFR) elegantly and simply describes the effect of epicardial obstruction in relation to a theoretical absence of this obstruction using the formula $\mathrm{Pd}-\mathrm{Ra} / \mathrm{Pa}-\mathrm{Ra}$, where $\mathrm{Pd}$ is the distal coronary pressure, $\mathrm{Pa}$ the aortic pressure and Ra the right atrial pressure. It is
Heart 2007;93:419-422. doi: 10.1136/hrt.2005.066837

extremely simple to measure using a pressure wire and is largely unaffected by haemodynamic conditions. In addition, there is an established cut-off value enabling easy decision making at the time of cardiac catheterisation.

In large studies, an FFR value $<0.75$ has been determined to be predictive of functional significance and, in addition, is the threshold at which revascularisation should be considered. ${ }^{2-4}$ However, it is absolutely crucial to have a clear understanding of the potential limitations of this measurement as incorrect interpretation may have dramatic implications with respect to the management strategy. Similarly, it is important to appreciate areas in which data supporting this cut-off value are less robust.

This article discusses issues regarding the optimal measurement of FFR, its potential pitfalls and situations where caution should be exercised in interpretation.

\section{CHOICE OF EQUIPMENT}

Pressure-wire studies should only be performed using guide catheters, not only because of the superior luminal characteristics of the catheter but also to allow rapid access with balloons or stents in the rare event of vessel dissection or perforation. Generally 6 or 7F catheters are recommended, if larger catheters are used, care should be taken to disengage from the coronary ostium when measurements are taken to avoid pressure damping, which could result in a falsely high calculated FFR. The use of side-hole catheters is possible, but only if intravenous rather than intracoronary vasodilators are used..$^{5}$

\section{VASODILATORS AND HYPERAEMIA}

Central to the mathematical derivation of the FFR using pressure measurements is the fact that the microvascular resistance remains minimal and constant in the presence or absence of an epicardial obstruction. The only condition where this is assumed to be the case is at maximum hyperaemia, although there remains some controversy in this regard. Therefore, maximal hyperaemia is crucial to FFR measurement as submaximal vasodilatation will reduce any translesion gradient and thus provide a falsely increased FFR value.

Hyperaemia may be induced as a result of ischaemia, but this is unreliable and it has been clearly shown that a further drop in microvascular resistance may be achieved by pharmacological means. The drug of choice for FFR measurements

Abbreviations: BCIS, British Cardiovascular Intervention Society; FFR, fractional flow reserve 


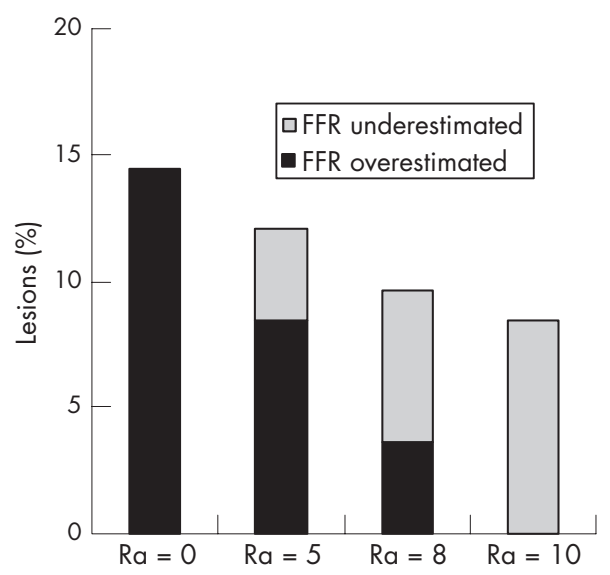

Figure 1 Misclassification of lesions for a functional flow reserve (FFR) threshold of 0.75 when right atrial $(\mathrm{Ra})$ pressure is assumed rather than measured.

is generally adenosine, although there has been much debate as to whether this should be administered through intracoronary injections or intravenous infusion.

Responses to different doses of adenosine have been examined. Intravenous administration of adenosine at a dose of $140 \mu \mathrm{g} / \mathrm{kg} / \mathrm{min}$ is believed to achieve maximum hyperaemia, and increasing the dose beyond this has not been shown to have any further effect on microvascular resistance. ${ }^{6}$ Some debate exists as to the optimum dose of intracoronary adenosine; doses of 15-20 $\mu \mathrm{g}$ in the right coronary artery and $18-24 \mu \mathrm{g}$ in the left coronary artery are generally recommended, although there is some evidence that higher doses may be required in some patients to truly achieve maximum pharmacological hyperaemia. ${ }^{7}$ In addition, the short half-life of adenosine provides only a brief window of maximum hyperaemia during which time measurements may be taken.

Papaverine (20 mg intracoronary (ic)), although an excellent vasodilatory stimulus, has been shown to provoke ventricular arrhythmias rarely and is therefore used infrequently. ${ }^{8}$ Likewise, sodium nitroprusside has been shown to be a potent vasodilatory stimulus and is not terribly user friendly, but may be a candidate in the presence of severe obstructive airway disease or severe conduction disease.

In our experience, the use of intravenous adenosine gives more consistent results. We have found that using the average measurements of aortic and distal coronary pressure obtained over several cardiac cycles provides a more reliable value for FFR than using the minimum values after bolus injection (unpublished data), and this is not possible when using bolus intracoronary adenosine because of the short half-life of the drug.

Leaving aside the argument as to how best to achieve optimum hyperaemia, the use of intravenous adenosine infusion facilitates pullback assessment of the coronary artery, which is key to assessing vessels with diffuse disease or the effect of percutaneous intervention as will be discussed later.

\section{RIGHT ATRIAL PRESSURE}

It has been argued by some that when calculating the FFR, right atrial pressure can be ignored and assumed to be zero unless it is clinically suspected to be markedly abnormal. We have examined the FFR in 62 patients with angiographically intermediate stenoses and recalculated the value on the basis of an assumed right atrial pressure of 0, 5, 8 and $10 \mathrm{~mm} \mathrm{Hg}$. In all, $14 \%$ of the lesions were found to be misclassified as insignificant when right atrial pressure was ignored (fig 1). ${ }^{9}$ In view of this, it is our practice to routinely measure right atrial pressure, and placement of a femoral venous sheath has the added advantage of providing central venous access for adenosine infusion. The value given for FFR on the console for the RADI wire (RADI Medical systems, Uppsala, Sweden) is in fact derived from distal coronary pressure and aortic pressure, ignoring right atrial pressure. The manufacturer maintains that for a value between 0.75 and 0.85 , clinical criteria should be used to determine the appropriateness of intervention. However, we argue that reducing the sensitivity and specificity to the level of non-invasive tests for ischaemia by introducing unnecessary inaccuracies is inappropriate, given that it takes only a matter of minutes to set up an additional transducer and place a right atrial line.

\section{SERIAL STENOSES/DIFFUSE DISEASE}

The FFR provides a specific index of the effect of intervention on an individual stenosis. However, when the pressure wire is placed in the distal coronary artery, this measurement reflects the pressure drop of the entire vessel and the component lesions thereof. It will inform us whether the total epicardial obstruction in the context of microvascular resistance and collateral flow is the cause of ischaemia and the potential gain of epicardial revascularisation. However, it is not possible to dissect out the relative contribution of individual lesions to the overall pressure drop without direct measurement of coronary wedge pressure, as the FFR does not reflect the sum of the pressures assessed at different points in the vessel. ${ }^{10}$ In the presence of serial stenoses, a distal lesion will artificially reduce the pressure gradient across the proximal lesion, leading to an overestimation of the proximal lesion's distal coronary pressure/aortic pressure ratio. Conversely, the presence of a proximal lesion will artificially lower this ratio for a distal lesion (fig 2). Correction of this coronary wedge pressure and coronary pressure between lesions is required to predict the FFR for individual stenoses necessitating coronary intervention. However, even if the wedge pressure is known and individual lesion FFR is calculated, ${ }^{11}$ the presence of side branches may introduce steal and thus render the measurement inaccurate.

\section{LEFT MAIN STEM DISEASE}

It has been established that those with haemodynamically relevant left main stem stenoses benefit from revascularisation on prognostic grounds. That said, there are frequently angiographically equivocal lesions which may present a management problem, in terms of either determining an appropriate revascularisation strategy or indeed whether to intervene at all. Intravascular ultrasound undoubtedly provides useful information, but if the ultrasound sample is not perpendicular to the vessel or there is a markedly eccentric lesion the luminal dimensions may be misleading.

The use of the pressure wire in this context is potentially valuable, especially in isolated left main stem lesions. Data have confirmed that the cut-off value of 0.75 is applicable to this lesion subset. ${ }^{11}$ However, in the presence of additional disease the serial stenosis principle outlined earlier comes into play; thus, interrogation of the left main stem lesion may only be performed if the pressure wire can be placed in the distal portion of an unobstructed vessel.

\section{MICROVASCULAR DISEASE AND LEFT VENTRICULAR DYSFUNCTION}

A number of conditions are known to be associated with microvascular abnormalities-for example, hypertension, left ventricular hypertrophy, left ventricular dysfunction, diabetes and hyperlipidaemia. Abnormal microvascular perfusion may arise from two mechanisms. Firstly, there may be reduced 


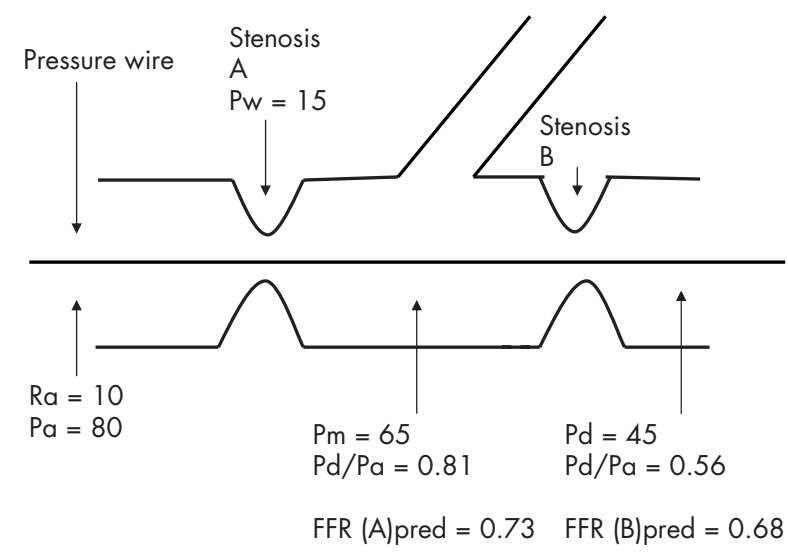

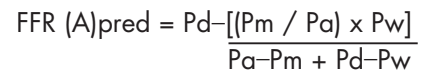

FFR (B)pred $=1-\frac{(\mathrm{Pa}-\mathrm{Pw}) \times(\mathrm{Pm}-\mathrm{Pd})}{\mathrm{Pa}(\mathrm{Pm}-\mathrm{Pw})}$

Figure 2 Fractional flow reserve (FFR) in the presence of serial stenoses in a diffusely diseased vessel. Use of distal coronary pressure/aortic pressure ratio is a poor surrogate for the functional significance of individual lesions in this context and, instead, calculation should be based on the above equations, which incorporate coronary wedge pressure at vessel occlusion as well as the pressure measurement between stenoses. $\mathrm{Pa}$, aortic pressure; $\mathrm{Pd}$, distal coronary pressure; Pm, pressure between stenoses, pred, predicted; Pw, coronary wedge pressure; Ra, right atrial pressure.

capillary density and, secondly, there may be abnormal vasoreactivity as a result of endothelial dysfunction. ${ }^{12}$

Much of the original validation data pertaining to FFR were obtained in patients with normal left ventricular function and did not specifically examine subgroups with microvascular abnormalities; indeed, in some studies these patients were actively excluded. ${ }^{2-4}$

In the case of left ventricular hypertrophy and diabetes, a lower ischaemic threshold is observed as a result of the relatively inadequate capillary supply for the muscle mass. ${ }^{13}$ In addition, in left ventricular hypertrophy an increased perfusion gradient is noted between sub-epicardial and subendocardial layers, increasing the propensity for transmural steal. ${ }^{12}$

It is therefore highly possible that in these conditions ischaemia will be present with lesser degrees of epicardial obstruction, and therefore an FFR cut-off value of 0.75 may be inappropriately low.

Similarly, the effect of left ventricular dysfunction on coronary pressure measurements is unclear. Impaired systolic function may be categorised into ischaemic and non-ischaemic forms, although data for both categories with respect to coronary physiology are limited.

It has been shown using positron emission tomography and echocardiographic techniques that myocardial blood flow reserve is abnormal in patients with idiopathic dilated cardiomyopathy. ${ }^{14}{ }^{15}$ The aetiology of impaired flow reserve is perhaps multifactorial and influenced by coronary perfusion pressure, vasomotor control, increased wall stress and increased extravascular compressive forces.

It is unclear exactly how the abnormal vasoreactivity observed in patients with microvascular dysfunction or impaired left ventricular systolic function will affect the validity of the FFR measurement. In theory, failure to achieve maximum hyperaemia as a result of potent vasoconstrictive mechanisms would lead to an underestimation of the translesion gradient with an "inappropriately high" FFR. However, part of the elegance of the FFR measurement is that it provides an assessment of the effect of relieving an epicardial obstruction, which in turn is influenced by microvascular resistance. In patients with abnormally high microvascular resistance, the benefit of relieving epicardial obstruction is relatively less and therefore the FFR value may not be "inappropriately high" with respect to the decision to intervene on a lesion.

Further studies specifically validating pressure-wire assessment in these subgroups with established non-invasive techniques are needed to deal with these issues. From a clinical point of view, in patients with potential microvascular dysfunction or poor left ventricular function, borderline FFR values should be interpreted with caution and management strategies should be guided not only by the pressure-wire assessment but also by symptoms and possibly supplementary non-invasive tests.

\section{ACUTE CORONARY SYNDROMES/MYOCARDIAL INFARCTION}

In the context of acute myocardial infarction, microvascular abnormalities prevail. Vessel obstruction occurs not only as a result of embolisation of thrombotic material, plaque debris and white cell and platelet aggregates but also as a result of endothelial swelling with luminal encroachment and potent vasoconstriction. Clinically this is apparent if there is sluggish clearance of contrast and failure of resolution of ST segment abnormalities despite epicardial vessel patency. In this context, microvascular resistance will be high and thus any translesional gradient diminished. Therefore, in the setting of acute myocardial infarction, the FFR value may be meaningless and entirely different from that obtained for the same lesion at a time distant from the acute event. In the context of acute myocardial infarction, the aim of primary or rescue percutaneous intervention is to restore vessel patency. Beyond this, intervention is often performed in intermediate lesions, not with the primary aim of ameliorating potential ischaemic symptoms but instead because of perceived "plaque stabilisation" as a result of stenting. Thus, the pressure wire may be of less value in this setting.

It remains a matter of debate as to whether complete revascularisation should be performed in the context of acute myocardial infarction. However, interrogation of non-target, intermediate lesions using the pressure wire at this time is 
potentially inaccurate, as abnormalities of microvascular function have been shown to extend to all arterial territories. ${ }^{16}$

With respect to prior myocardial infarction ( $\geqslant 6$ days), it is believed that microvascular resistance in the infarcted territory is inversely proportional to the amount of viable myocardium, and thus FFR measurements to determine the functional significance of an epicardial lesion remain valid, with a cut-off of 0.75 ; however the sensitivity and specificity of measurements are slightly less than for non-infarcted myocardium. ${ }^{17} 18$

Few data are available with respect to non-ST-segment elevation infarction or unstable angina and physiological measurements. These prothrombotic, proinflammatory conditions are known to affect the microvasculature, albeit less dramatically than ST segment elevation infarction; however, again it is unclear to what degree this affects the validity of the FFR measurement and is likely to vary from individual to individual.

\section{Post-interventional FFR}

It has been shown that a post-interventional FFR $>0.9$ is associated with superior long-term outcomes and a reduced need for repeat revascularisation. ${ }^{19}$

Hypothetically, the reasons for a low FFR after intervention may be threefold. Firstly, there may be residual obstruction at the area of intervention - that is, suboptimal stent deployment. Secondly, there may be diffuse disease throughout the epicardial vessel. In this situation, performing a pullback of the pressure wire will distinguish between discrete obstruction, in which there will be an abrupt step-up in pressure, and diffuse disease, where a gradual increase in pressure will be observed. Finally, reactive hyperaemia has been described after coronary intervention. ${ }^{12}$ It is unclear whether this is a more profound hyperaemia than that achieved pharmacologically, but if so it may exacerbate any residual pressure drop in the vessel.

If a suboptimal post-interventional FFR is the result of inadequate treatment of the original lesion, post-dilatation or additional stenting may be appropriately used. However, it should be noted that the data with respect to postinterventional FFR and prognosis refer to target vessel revascularisation rather than target lesion revascularisation, which may have implications in diffusely diseased vessels; furthermore, these studies were performed in the bare metal stent era. No data are available to clarify what should be done if diffuse disease is present; however, some may argue that in the drug-eluting stent era there may be a role for greater vessel coverage to eliminate diffuse, albeit subcritical, obstruction. It is possible that aggressive secondary prevention with statins and ACE inhibitors may be a more appropriate strategy in this situation.

\section{CONCLUSION}

The pressure wire is immensely useful in current interventional practice and it has provided invaluable insights into coronary physiology. The success of this technique lies in its simplicity, but it is crucial that such simplicity does not blind the operator of the limitations of the FFR or the scope of supporting data.
Similarly, although the generally robust cut-off value of 0.75 allows decisions to be made confidently regarding revascularisation, it is imperative that measurements are taken meticulously to ensure that the value obtained for an individual is truly representative.

\section{Authors' affiliations}

Lucy J Blows, Simon R Redwood, Cardiothoracic Unit, St Thomas'

Hospital, London, UK

Competing interests: None declared.

\section{REFERENCES}

1 Spaan JA, Piek JJ, Hoffman Jl, et al. Physiological basis of clinically used coronary hemodynamic indices. Circulation 2006;113:446-55.

2 Pijls NH, de Bruyne B, Peels K, et al. Measurement of fractional flow reserve to assess the functional severity of coronary-artery stenoses. N Engl J Med 1996:334:1703-8.

3 Chamuleau SA, Meuwissen M, Koch KT, et al. Usefulness of fractional flow reserve for risk stratification of patients with multivessel coronary artery disease and an intermediate stenosis. Am J Cardiol 2002;89:377-80.

4 Bech GJ, de Bruyne B, Bonnier HJ, et al. Long-term follow-up after deferral of percutaneous transluminal coronary angioplasty of intermediate stenosis on the basis of coronary pressure measurement. J Am Coll Cardiol 1998;31:841-7.

5 de Bruyne B, Stockbroeckx J, Demoor D, et al. Role of side holes in guide catheters: observations on coronary pressure and flow. Cathet Cardiovasc Diagn 1994;33:145-52

6 de Bruyne B, Pijls $\mathrm{NH}$, Barbato $\mathrm{E}$, et al. Intracoronary and intravenous adenosine 5 '-triphosphate, adenosine, papaverine, and contrast medium to assess fractional flow reserve in humans. Circulation 2003;107:1877-83.

7 Lopez-Palop R, Saura D, Pinar E, et al. Adequate intracoronary adenosine doses to achieve maximum hyperaemia in coronary functional studies by pressure derived fractional flow reserve: a dose response study. Heart 2004;90:95-6.

8 Hau WK. Fractional flow reserve and complex coronary pathologic conditions. Eur Heart J 2004:25:723-7.

9 Perera D, Biggart S, Postema P, et al. Right atrial pressure: can it be ignored when calculating fractional flow reserve and collateral flow index? J Am Coll Cardiol 2004;44:2089-91.

10 de Bruyne B, Pijls NH, Heyndrickx GR, et al. Pressure-derived fractional flow reserve to assess serial epicardial stenoses: theoretical basis and animal validation. Circulation 2000;101:1840-7.

11 Bech GJ, Droste H, Pijls NH, et al. Value of fractional flow reserve in making decisions about bypass surgery for equivocal left main coronary artery disease. Heart 2001;86:547-52.

12 Komaru T, Kanatsuka H, Shirato K. Coronary microcirculation: physiology and pharmacology. Pharmacol Ther 2000;86:217-61.

13 Krams R, Kofflard MJ, Duncker DJ, et al. Decreased coronary flow reserve in hypertrophic cardiomyopathy is related to remodeling of the coronary microcirculation. Circulation 1998:97:230-3.

14 van den Heuvel AF, van Veldhuisen DJ, van der Wall EE, et al. Regiona myocardial blood flow reserve impairment and metabolic changes suggesting myocardial ischemia in patients with idiopathic dilated cardiomyopathy. J Am Coll Cardiol 2000:35:19-28.

15 Vanderheyden M, Bartunek J, Verstreken S, et al. Non-invasive assessment of coronary flow reserve in idiopathic dilated cardiomyopathy: hemodynamic correlations. Eur J Echocardiogr 2005;6:47-53.

16 Kyriakides ZS, Antoniadis A, Kolettis TM, et al. Coronary flow reserve in the contralateral artery increases after successful coronary angioplasty in patients with spontaneously visible collateral vessels. Heart 1998;80:493-8.

17 de Bruyne B, Piils NH, Bartunek J, et al. Fractional flow reserve in patients with prior myocardial infarction. Circulation 2001;104:157-62.

18 Usui Y, Chikamori T, Yanagisawa H, et al. Reliability of pressure-derived myocardial fractional flow reserve in assessing coronary artery stenosis in patients with previous myocardial infarction. Am J Cardiol 2003;92:699-702

19 Pijls NH, Klauss V, Siebert U, et al. Coronary pressure measurement after stenting predicts adverse events at follow-up: a multicenter registry. Circulation 2002;105:2950-4. 